\title{
Vitamin D as a Mediator of Racial Differences in Blood Pressure
}

\author{
R. Neal Axon, $M D, M S C R^{1,2}$, Jeffrey A. Tice, $M D^{3}$, and Leonard E. Egede, MD, MS $1,2,4$ \\ ${ }^{1}$ Center for Disease Prevention and Health Interventions for Diverse Populations, Ralph H. Johnson VAMC, Charleston, SC, USA; ${ }^{2}$ Department \\ of Medicine, Division of General Internal Medicine and Geriatrics, The Medical University of South Carolina, Charleston, SC, USA; ${ }^{3}$ Department \\ of Medicine, Division of General Internal Medicine, The University of California at San Francisco, San Francisco, CA, USA; ${ }^{4}$ Center for Health \\ Disparities Research, Medical University of South Carolina, Charleston, SC, USA.
}

J Gen Intern Med 26(10):1088-9

DOI: $10.1007 / \mathrm{s} 11606-011-1791-9$

(c) Society of General Internal Medicine 2011

$\mathrm{H}$ ypertension is a cardinal risk factor for myocardial infarction, congestive heart failure, stroke, and death. Despite unfavorable trends in the prevalence of diabetes, obesity, and sedentary lifestyles, overall hypertension prevalence was stable at $\sim 29 \%$ between the 1999-2000 and 20072008 time periods $^{1,2}$. This phenomenon has been attributed to improved hypertension awareness, higher treatment rates, and more intense regimens for hypertension. Unfortunately, overall hypertension prevalence among black Americans remains close to $40 \%$, significantly higher than the level observed for whites and Hispanic groups ${ }^{2,3}$. Similar patterns of persistent racial differences despite overall improvement have been observed in cross-sectional studies of blood glucose levels and cholesterol levels ${ }^{4}$. Longitudinal studies also show increased odds of poor blood pressure control over time among Non-Hispanic blacks compared to whites ${ }^{5}$. In addition to increased prevalence of cardiovascular risk factors, mortality from coronary heart disease and stroke remain higher for blacks than other racial/ethnic groups ${ }^{6}$. Therefore, substantial interest exists to explain these persistent disparities and to devise interventions that can address them.

In this issue of the Journal, Fiscella and colleagues present evidence that vitamin D deficiency is a potential mediator of racial differences in blood pressure between blacks and whites $^{7}$. In an analysis of NHANES data from 2001-2006, they demonstrated that 25-hydroxyvitamin D (25-OH D) levels appeared to account for approximately one quarter of the difference in mean systolic blood pressure observed between black and white subjects. This study is notable because it is the largest and most robust to date to examine the effect of Vitamin D on racial disparities in blood pressure. The analysis is strengthened by a relatively large sample size with adequate power to detect differences between races based on vitamin $\mathrm{D}$ levels. The investigators also extensively adjusted for potential confounders of the relationship between black and white blood pressure measures.

In addition to known effects on bone metabolism, Vitamin D has been the subject of intense investigation in recent years given interest in its other systemic effects. Observational studies have associated low vitamin D levels with increased

Published online July 6, 2011 risk for cancer, autoimmune diseases, diabetes, and cardiovascular disease including hypertension ${ }^{8-11}$. A role for vitamin $\mathrm{D}$ in cardiovascular disease is plausible given demonstrated effects on parathyroid hormone levels, renin-angiotensin activation, and vascular reactivity ${ }^{12}$. This effect may be more pronounced among blacks because their darker skin tone, high prevalence of lactase deficiency, and lower dietary intake of dairy products ${ }^{13}$ increases their risk for low vitamin D. Further, observational studies have demonstrated an association between low vitamin D levels and hypertension. For example, in one meta-analysis the pooled relative risk of incident hypertension over 8 years was 1.76 (95\% CI 1.27, 2.44) when comparing individuals in the highest and lowest vitamin D concentrations ${ }^{9}$.

While the findings in the present study are provocative, care should be taken in their interpretation. First, the crosssectional nature of the analysis precludes causal inferences. Though the investigators adjusted for key socio-demographic factors, patient-specific health conditions/behaviors, and biomarkers; the potential for bias and confounding remains. For example, information on psychosocial stress, medication non-adherence, and health literacy were not available for inclusion in this analysis. These factors have previously been implicated as mediators of racial blood pressure differences ${ }^{14}$. Information is also lacking on seasonal variation in vitamin $\mathrm{D}$ levels and diurnal variation in blood pressure. Finally, diet and lifestyle factors such as sodium intake, physical activity, and sun exposure may have contributed to the observed differences in blood pressure.

Even if vitamin D deficiency does explain a portion of the black-white blood pressure disparity, we must consider the present study within the context of the randomized clinical trials that have failed to demonstrate a benefit of vitamin D supplementation for the primary or secondary prevention of hypertension. Pittas and colleagues systematically reviewed 17 clinical trials of vitamin D supplementation, and all but two showed no significant effect of vitamin D supplementation on systolic or diastolic blood pressure ${ }^{9}$. Most studies to date have been small with fewer than 100 participants, but the study of calcium and vitamin D supplementation conducted as part of the Women's Health Initiative (WHI) was by far the largest with over 36,000 women randomized to $1000 \mathrm{mg}$ elemental calcium and 400 IU of vitamin D3 daily versus placebo ${ }^{15}$. After an average of 7 years' follow up, there were no differences in incident hypertension or the change over time in systolic or diastolic blood pressure between intervention and control patients. These results were consistent in subset analyses stratified by race, baseline vitamin D levels, and the presence or absence of baseline hypertension. 
Critics have argued that the WHI study had some limitations including recruiting a sample of relatively healthy volunteers with low levels of vitamin D deficiency at baseline (25\%), allowing for open label vitamin D supplementation (up to 600 IU per/day) which may have contributed to contamination between groups and among participants with baseline vitamin D deficiency, and that the dose of vitamin D used in the study may have been too low to raise bodily levels above a therapeutic threshold ${ }^{16}$. In spite of these criticisms, the lack of association in such a large sample followed over 7 years suggests that vitamin D supplementation does not affect blood pressure in blacks or patients with low baseline vitamin D levels. The only biologically plausible issue appears to be whether long-term low vitamin D status leads to irreversible changes in the vasculature that cause hypertension.

Clearly, the issue of vitamin D and its effects on blood pressure requires further research. However, current evidence suggests that vitamin D supplementation does not affect blood pressure. One recent study ${ }^{17}$ randomized 438 subjects to either 0,20,000 or 40,000 IU of vitamin D3 per week for a year and all subjects were given $500 \mathrm{mg}$ calcium daily. The study showed significant increases in vitamin D levels, but no significant effect on blood pressure. The WHI analyses adequately addressed the issue of whether there is effect modification, interaction or differential effects in the subgroups defined by low initial vitamin D level or among blacks. Although the evidence is not perfect, it is pretty strong that vitamin D supplementation does not affect blood pressure and that the association between vitamin $\mathrm{D}$ and blood pressure is another great example of either the ecological fallacy (most likely) or unknown confounders or residual confounding from incomplete measurement of the confounders. More studies are needed about the specific effect of vitamin D deficiency on blood pressure among patients with and without known hypertension. This might be obtained through pooled analysis of existing randomized blood pressure medication trials with available stored blood samples. Analyses could determine if baseline vitamin $\mathrm{D}$ deficiency modulates blood pressure outcomes among treated patients in comparison to controls. In addition further prospective randomized trials are needed that avoid concerns of patient selection by focusing solely on black patients or perhaps more specifically on individuals with low vitamin D levels. Such trials should carefully restrict and monitor open-label vitamin D supplementation, dietary intake, and sun exposure to control for inter-group contamination. Finally, future trials should utilize higher doses of vitamin D sufficient to fully treat deficiency states.

If this line of research is further validated, then additional measures may be warranted at the population level. For example, should the FDA and other organizations develop race-specific recommendations for dietary vitamin D? Should vitamin $\mathrm{D}$ be used to fortify additional food items besides milk and orange juice? In contrast to other food additives, the overall risk of vitamin D intoxication from such measures would be low. In light of evolving knowledge on the systemic effects of vitamin $\mathrm{D}$, there might be additional benefits to better population-level approaches to vitamin D supplementation beyond cardiovascular and bone effects. For now, additional research is needed to better characterize the relationship between vitamin $\mathrm{D}$ and blood pressure across racial/ethnic groups and other relevant subgroups.

Corresponding Author: Leonard E. Egede, MD, MS; Center for Health Disparities Research, Medical University of South Carolina, 135 Rutledge Avenue, Room 280H, P.O. Box 250593, Charleston, SC 29425-0593, USA (e-mail: egedel@musc.edu).

\section{REFERENCES}

1. Hajjar I, Kotchen TA. Trends in prevalence, awareness, treatment, and control of hypertension in the United States, 1988-2000. JAMA. 2003;290(2): 199-206.

2. Egan BM, Zhao Y, Axon RN. US trends in prevalence, awareness, treatment, and control of hypertension, 1988-2008. JAMA. 2010;303 (20):2043-2050.

3. Ostchega Y, Hughes JP, Wright JD, McDowell MA, Louis T. Are demographic characteristics, health care access and utilization, and comorbid conditions associated with hypertension among US adults? Am J Hypertens. 2008;21(2):159-165.

4. McWilliams JM, Meara E, Zaslavsky AM, Ayanian JZ. Differences in control of cardiovascular disease and diabetes by race, ethnicity, and education: U.S. trends from 1999 to 2006 and effects of medicare coverage. Ann Intern Med. 2009;150(8):505-515.

5. Axon RN, Gebregziabher M, Echols C, Gilbert GE, Egede LE. Racia and Ethnic Differences in Longitudinal Blood Pressure Control in Veterans with Type 2 Diabetes Mellitus. J Gen Intern Med. 2011. In Press.

6. Keenan NL, Shaw KM. Coronary heart disease and stroke deaths United States, 2006. MMWR Surveill Summ. 2011;60(Suppl):62-66.

7. Fiscella K, Winters P, Tancredi D, Franks P. Racial disparity in blood pressure: Is vitamin D a factor? J Gen Intern Med. doi:10.1007/s11606011-1707-8.

8. Garland CF, Garland FC, Gorham ED, Lipkin M, Newmark H, Mohr SB, et al. The role of vitamin D in cancer prevention. Am J Publ Health. 2006;96(2):252-261.

9. Pittas AG, Chung M, Trikalinos T, Mitri J, Brendel M, Patel K, et al Systematic review: Vitamin D and cardiometabolic outcomes. Ann Intern Med. 2010;152(5):307-314.

10. Munger KL, Levin LI, Hollis BW, Howard NS, Ascherio A. Serum 25hydroxyvitamin D levels and risk of multiple sclerosis. JAMA. 2006;296 (23):2832-2838.

11. Hypponen E, Laara E, Reunanen A, Jarvelin MR, Virtanen SM. Intake of vitamin D and risk of type 1 diabetes: a birth-cohort study. Lancet. 2001;358(9292):1500-1503.

12. Holick MF. Vitamin D. deficiency. New Engl J Med. 2007;357(3):266281.

13. Fulgoni V 3rd, Nicholls J, Reed A, Buckley R, Kafer $\mathbf{K}$, Huth P, et al. Dairy consumption and related nutrient intake in African-American adults and children in the United States: continuing survey of food intakes by individuals 1994-1996, 1998, and the National Health And Nutrition Examination Survey 1999-2000. J Am Diet Assoc. 2007;107 (2):256-264.

14. Bosworth HB, Dudley T, Olsen MK, Voils CI, Powers B, Goldstein MK, et al. Racial differences in blood pressure control: potential explanatory factors. Am J Med. 2006;119(1):70. e9-15.

15. Margolis KL, Ray RM, Van Horn L, Manson JE, Allison MA, Black HR, et al. Effect of calcium and vitamin D supplementation on blood pressure: the Women's Health Initiative Randomized Trial. Hypertension. 2008;52(5):847-855.

16. Geleijnse JM. Vitamin D. and hypertension: does the women's health initiative solve the question? Hypertension. 2008;52(5):803-804.

17. Jorde R, Sneve $\mathbf{M}$, Torjesen $\mathbf{P}$, Figenschau $\mathbf{Y}$. No improvement in cardiovascular risk factors in overweight and obese subjects after supplementation with vitamin D3 for 1 year. J Intern Med. 2010;267 (5):462-472. 